Ann. Biol. anim. Bioch. Biophys., I975, 15 (I), 37-47.

\title{
MÉTHODES RADIOISOTOPIQUES D'ÉTUDE DE LA PÉNÉTRATION GUTANÉE DES IONS
}

\author{
G. MELKI \\ Laboratoire de Physique médicale, \\ Faculté de Médecine, Boulevard du Professeur L.-Bernard, \\ 35000 Rennes \\ RÉSUMÉ
}

L'absorption des ions à travers la peau du jeune Rat, baigné dans une solution radioactive, a été étudiée par mesure de l'activité de certains organes rapportée à l'activité du bain, d'une part, et par celle de l'activité totale du corps de l'animal écorché de son revêtement cutané et introduit dans le cristal puits, d'autre part. La pénétration de l'iode est plus importante que celle du sodium et du potassium et dure plus longtemps. Chez l'Homme, l'application de pansements antébrachiaux occlusifs a permis de démontrer l'absorption percutanée de divers ions, dont l'iodure et le pertechnetate; enfin, la diminution du taux de fixation thyroiddienne d'iode I3I après application cutanée de préparation commerciale contenant de l'iode froid ou de la thyroxine fournit une méthode indirecte mais sensible de mise en évidence du phénomène de pénétration percutanée chez l'Homme; cette méthode constitue également un moyen d'étude de l'action de différents paramètres physiques susceptibles d'influencer l'absorption percutanée de l'iode ou de l'iodure.

L'intérêt de la connaissance de la pénétration cutanée des ions réside à la fois dans son application thérapeutique et l'usage de la balnéothérapie.

\section{INTRODUCTION}

I a voie transcutanée est utilisée couramment en thérapeutique pour introduire dans l'organisme un certain nombre de composés médicamenteux. Nombreux sont les travaux qui traitent du passage de solvants organiques, excipients, hormones, vitamines $[2,5,7, \mathrm{II}, \mathrm{I} 6, \mathrm{I} 7]$. Plus rares sont les auteurs qui se sont intéressés à la pénétration cutanée d'électrolytes en solution aqueuse [IO, I2, I5, 20], pénétration qui présente néanmoins un intérêt considérable en matière de thalassothérapie $[4,8]$. Le passage de molécules d'eau à travers la peau du rat a été démontré grâce 
à l'emploi d'eau tritiée ou deutériée [9]. Ce n'est que récemment que les expérimentateurs ont utilisé les isotopes radioactifs et la technique d'autohistoradiographie pour étudier la pénétration de molécules ou ions à travers la peau $\left[6, I_{3}, I_{4}, I_{5}, I_{8} 8\right.$, I9:.

Cependant, la variabilité des méthodes mises en jeu faisant appel à des animaux de laboratoires différents, à des substances variées et le mode d'expression même des données, rendent difficile la confrontation des résultats des divers auteurs. Les données expérimentales obtenues in vivo chez l'animal, aussi riches d'enseignement soient-elles, ne peuvent être, sans précautions, extrapolées à 1'Homme malgré les communautés de structure pouvant exister entre la peau humaine et celle de l'animal d'expérience.

BARTEK et al. [r] ont en effet montré, grâce à l'emploi de stéroïdes marqués, que la perméabilité de la peau humaine in vivo était plus voisine de celle du porcelet que celle du lapin ou du rat, tout en étant la plus faible des quatre.

DUBARRY et al. $[3,4]$ ont montré la complexité de la pénétration cutanée des ions et considérablement clarifié le problème en analysant soigneusement l'influence de nombreux paramètres physicochimiques.

Dans cette étude, nous avons testé in vivo, par la méthode radioisotopique, la perméabilité cutanée du jeune rat à divers ions puis essayé de démontrer chez l'Homme la réalité des phénomènes de pénétration transcutanée vis-à-vis d'ions couramment rencontrés dans l'eau de mer.

\section{MATÉRIEL E'T MÉTHODES}

Le schéma expérimental que nous avons adopté pour étudier la pénétration percutanée chez le jeune Rat est inspiré des travaux de Dubarry et al. $[3,4]$ avec cependant quelques variantes pour exprimer ce phénomène. Les ions émetteurs gamma utilisés ont été le sodium 24 , le potassium $4_{2}^{2}$, l'iode $13 \mathrm{I}$, le strontium 85 , le technétium $99 \mathrm{~m}$. Nous avons aussi procédé, parallèlement à l'expérimentation animale, à une étude sur des humains volontaires; deux procédés expérimentaux ont été employés, le premier conduisant à une mesure directe de la pénétration d'ions radioactifs, le second mettant en évidence, de façon indirecte, le passage de composés iodés stables.

\section{1. -- Pénétration des ions à travers la peau du jeune rat}

Des rats de 8 jours sont baignés dans une solution active contenue dans des flacons de $30 \mathrm{ml}$ environ. La tête et les pattes de devant restent hors du bain. Chaque rat est maintenu dans un flacon par une rondelle de caoutchouc qui l'enserre au-dessous des pattes de devant. La rondelle bloquée par une couronne métallique forme écran et prévient toute contamination rétrograde par voie aéro-digestive. Ce dispositif a l'avantage d'être peu traumatisant, ce qui permet des manipulations de longue durée. Nous n'avons observé aucun décès en cours de baignade.

La préparation radioactive est dissoute dans de l'eau distillée et répartie entre les flacons maintenus à $30^{\circ} \mathrm{C}$. La durée du bain varie de o à 5 heures.

En fin de baignade, le jeune rat est sacrifié puis lavé. Nous procédons alors soit :

- à une dissection que l'on commence par la partie antérieure non contaminée de l'animal ; le foie, la rate et la thyroïde sont prélevés. L'activité de ces organes est comptée au cristal-puits et comparée à l'activité de l'unité de volume ( $1 \mathrm{ml}$ ) du bain ;

- à un dépouillement de l'animal de haut en bas de son revêtement cutané en retroussant de dedans en dehors la peau, ce qui élimine toute la radioactivité cutanée ; le corps du jeune rat est alors placé dans un doigtier de caoutchouc qui peut être introduit dans le cristal-puits pour 
mesure de l'activité ayant pénétré dans l'organisme. L'activité trouvée est divisée par l'activité de $\mathrm{ml}$ du bain. Le rapport correspondant est un volume qui représente le volume en ml de solution radioactive épurée de la quantité de traceur retrouvée dans le corps de l'animal.

$$
\text { 2. - Pénétration des ions à travers la peau de l'homme }
$$

\section{a) Premier protocole.}

Un tampon imbibé d'une solution contenant un radioisotope $\left({ }^{131} \mathrm{I},{ }^{85} \mathrm{Sr},{ }^{24} \mathrm{Na},{ }^{42} \mathrm{~K},{ }^{99 \mathrm{~m}} \mathrm{Tc}\right)$ est fixé sur l'avant-bras du sujet. Une enveloppe imperméable occlusive interdit toute fuite ou possibilité de contamination. L'activité du tampon est mesurée par comptage à distance. Au bout de I 2 heures, le tampon est enlevé. Les urines sont recueillies tout le temps de l'expérience. L'apparition d'une activité plasmatique, urinaire ou, le cas échéant, thyroïdienne témoigne du passage du traceur. A la fin du temps de contact, sont mesurées l'activité plasmatique, l'activité globale des urines et, le cas échéant, l'activité thyroïdienne. Le volume plasmatique nécessaire au calcul de l'activité plasmatique globale est déterminée par une méthode de dilution isotopique, au moyen d'albumine humaine radioiodée. Les valeurs obtenues sont rapportées à l'activité globale du tampon.

\section{b) Deuxième protocole.}

Il repose sur l'étude de la fixation d'iodure radioactif par le corps thyroïde. On sait qu'en exploration fonctionnelle thyroïdienne, une surcharge iodée est susceptible de perturber considérablement le test de fixation de l'iode radioactif. Il nous a paru intéressant de juger de l'importance du passage d'ions I- ou de composés iodés par la diminution éventuelle du taux de fixation thyroïdienne de l'iode I 31 .

Sur I9 volontaires, on effectue d'abord un test de fixation de l'iode I 3 I (ingestion de $25 \mu \mathrm{Ci}$ d'iode I3r). Dix jours plus tard, on applique sur la peau de l'avant-bras des sujets des préparations pharmaceutiques iodées non radioactives. On attend deux jours puis on effectue un deuxième test de fixation de l'iode r3I. Les composés testés contenaient, l'un : de l'iode (pommade Iodex), l'autre de la thyroxine (lotion Percutacrine thyroxinique dosée à $2 \mathrm{mmg}$ ).

\section{RÉSULTATS}

\section{A. - Chez le jeune Rat}

La pénétration dans l'organisme du $\mathrm{Na}$, du $\mathrm{K}$ et du $\mathrm{Sr}$ est d'abord rapide puis de plus en plus lente. Celle du sodium est plus importante que celle du potassium ou du strontium (fig. I).

En ce qui concerne l'iodure, on observe, dans des conditions expérimentales analogues, une pénétration relativement importante; l'iodure pénètre mieux que les autres ions étudiés. Par ailleurs, on peut remarquer (tabl. I) que pour un bain de trois heures la pénétration cutanée de l'iodure continue à crô̂tre sans saturation ni fléchissement, comme observé pour le potassium. Le tableau 2 rapporte les activités retrouvées dans le foie, la rate et la thyroïde de l'animal après quatre heures de bain. La radioactivité thyroïdienne est souvent inférieure à la radioactivité hépatique mais il est vraisemblable que la fixation thyroïdienne n'a pas atteint sa valeur en plateau au moment de la mesure.

Enfin, les essais menés avec de l'eau de mer révèlent une pénétration cutanée de l'iode inférieure à celle obtenue avec un bain d'eau distillée. Tout se passe donc comme si l'élévation de la force ionique du bain ralentissait le phénomène de pénétration percutanée, tout au moins dans le cas de l'iode. Ce résultat est en accord avec celui d'autres auteurs $[3,4]$. 


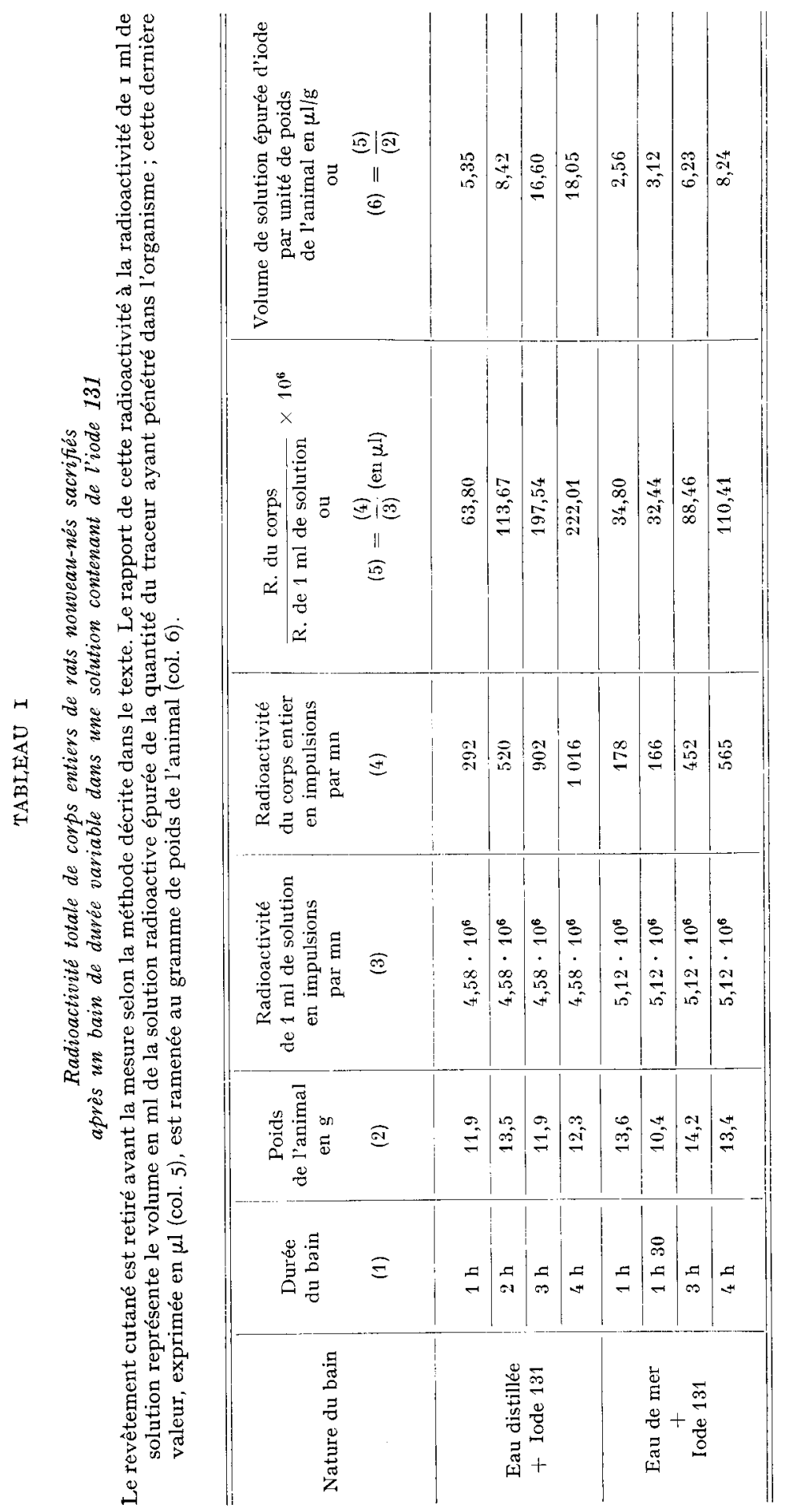




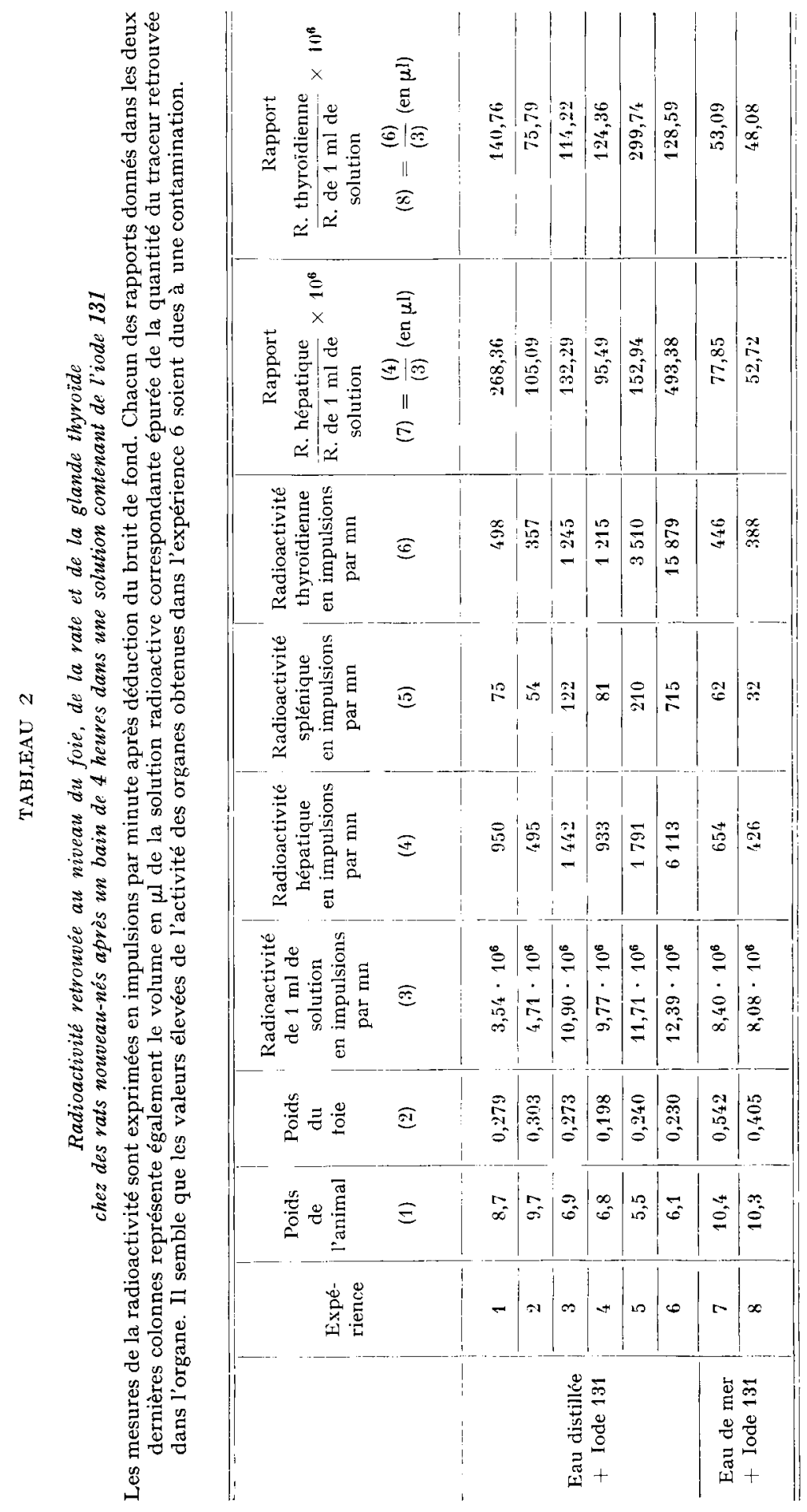




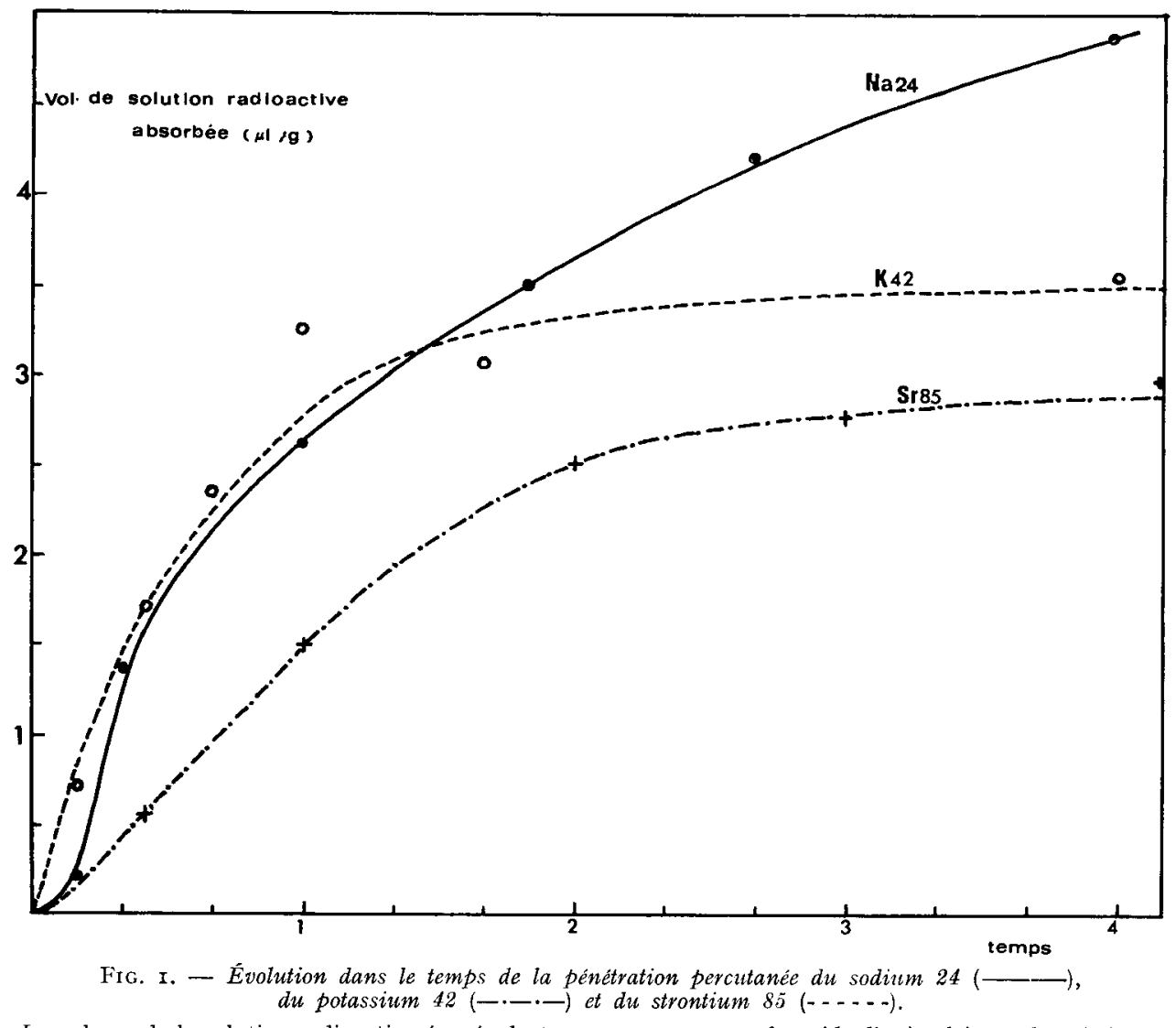

Le volume de la solution radioactive épurée du traceur par gramme de poids d'animal (en ordonnées) est représentée en fonction du temps du bain (en abscisses). Chaque point correspond à un animal différent.

\section{TABLEAU 3}

Pénétration du sodium 24 appliqué sous forme de pansement occlusif pendant 12 heures sur l'avant-bras de 5 sujets; l'activité plasmatique totale est rapportée à l'activité globale appliquée

\begin{tabular}{|c|c|c|c|c|c|}
\hline Sujet & $\begin{array}{c}\text { (1) } \\
9\end{array}$ & $\begin{array}{l}(2) \\
9\end{array}$ & $\begin{array}{c}(3) \\
?\end{array}$ & $\begin{array}{l}\left(\begin{array}{l}4 \\
)\end{array}\right) \\
?\end{array}$ & (5) \\
\hline Durée d'application & $24 \mathrm{~h}$ & $24 \mathrm{~h}$ & $24 \mathrm{~h}$ & $24 \mathrm{~h}$ & $24 \mathrm{~h}$ \\
\hline$\frac{\text { Activité plasmatique }}{\text { Activité appliquée }}$ & $2,12 \cdot 10^{-3}$ & $2,10 \cdot 10^{-3}$ & $0,6 \cdot 10^{-3}$ & $1,33 \cdot 10^{-\mathbf{3}}$ & 0 \\
\hline$\frac{\text { Activité urinaire }}{\text { Activité appliquée }}$ & $3,01 \cdot 10^{-3}$ & 0 & $1,07 \cdot 10^{-3}$ & $2,20 \cdot 10^{-3}$ & $\cdots$ \\
\hline
\end{tabular}




\title{
B. - Chez l'Homme
}

\section{Mesure directe de la pénétration.}

Les applications de compresses effectuées avec le sodium 24 (tabl. 3) nous amènent à observer qu'environ le millième environ de l'activité mise au contact est retrouvé dans le plasma après une application de I2 heures ; 1'analyse des urines recueillies confirme cet ordre de grandeur. Pour le potassium 42 , une pénétration transcutanée du même ordre de grandeur que pour le sodium est observée après I2 heures (tabl. 4). Dans tous les cas, l'activité restée au niveau de la compresse demeure très élevée et sa mesure ne rend pas compte de la pénétration.

\section{TABLEAU 4}

\begin{abstract}
Pénétration du potassium 42 appliqué sous forme de pansement occlusif pendant 12 heures sur l'avant-bras de 2 sujets; l'activité plasmatique totale est rapportée à l'activité globale appliquée
\end{abstract}

\begin{tabular}{c|c|c}
\hline Sujet & $(1)$ & $(2)$ \\
& $\delta$ & 9 \\
\hline Durée d'application & $12 \mathrm{~h}$ & $12 \mathrm{~h}$ \\
\hline Activité plasmatique & $1,42 \cdot 10^{-3}$ & $1,87 \cdot 10^{-3}$ \\
\hline Activité appliquée & $3,40 \cdot 10^{-3}$ & $2,65 \cdot 10^{-3}$ \\
\hline Activité urinaire & & \\
\hline Activité appliquée & & \\
\hline
\end{tabular}

Les comptages effectués en regard de la thyroïde, après application d'une compresse imbibée d'une solution d'iodure I3I, montrent une fixation par la glande qui s'accroît au cours du temps (tabl. 5). Les comptages sur le plasma n'ont pas donné de résultats significatifs mais la mesure de l'activité urinaire montre que la pénétration de l'iode est indiscutablement plus élevée que celle du sodium et du potassium et de l'ordre du I/Ioo de la radioactivité appliquée. Chez deux sujets, l'expérience a été renouvelée trois fois mais en utilisant pour chacune d'elles un solvant différent ; la préparation radioactive d'iodure de sodium a été fractionnée en trois parties égales : la première diluée dans de l'eau distillée, la deuxième dans du sérum physiologique et la troisième dans de l'eau de mer ; dans tous les cas, une pénétration équivalente d'iodure I3I fut retrouvée.

Le technétium 99m (sous forme de pertechnétate) a des propriétés chimiques voisines de celles de l'iode et possède une bonne affinité pour le tissu thyroïdien; en outre, il est d'une manipulation facile et peu dangereuse puisqu'il s'agit d'un isotope radioactif émetteur $\gamma$ de période physique brève ( 6 heures) et d'énergie relativement faible (I $40 \mathrm{keV}$ ). Malgré la brièveté du temps de contact, que l'on choisit égal à 6 heures, les comptages thyroïdiens et plasmatiques démontrent sa pénétration. 


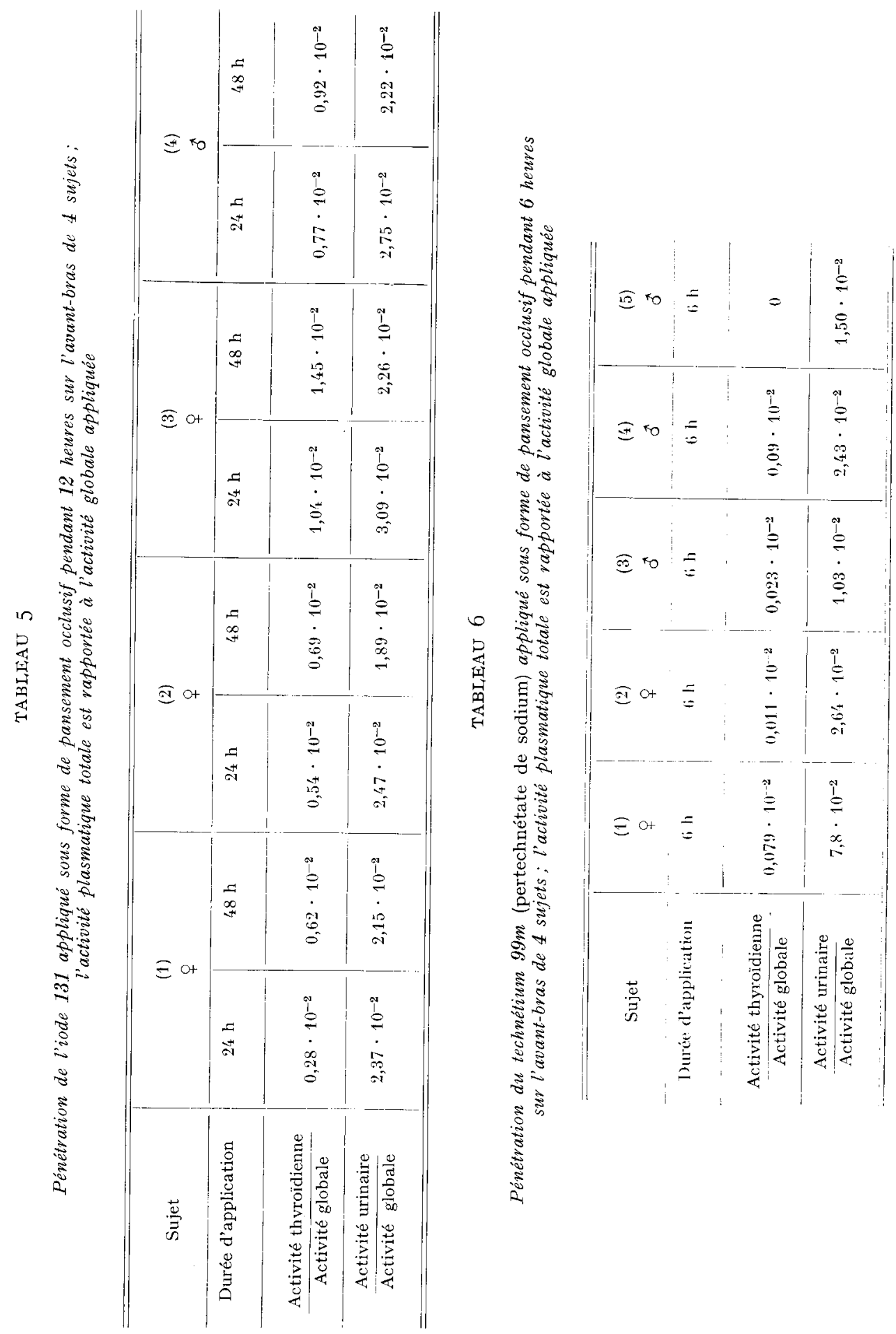


Si l'on compare les résultats obtenus avec l'iode I3I à ceux avec le technétium $99 \mathrm{~m}$ (tab1. 6), on constate que les pourcentages de fixation thyroïdienne sont inférieurs dans le cas du technétium cependant que les taux de radioactivité urinaire sont peu différents.

\section{Démonstration indirecte d'une pénétration transcutanée d'iode non radioactif.}

Une diminution presque constante du taux de fixation thyroïdienne de radioiodure chez un même sujet après applications de corps iodés peut être remarquée (fig. 2 et 3). Cette modification est due à l'enrichissement du pool extrathyroïdien d'iode secondaire à un phénomène de pénétration transcutanée dans l'intervalle des tests. A ce stade de nos résultats, nous pouvons dire que nous disposons là d'une méthode suffisamment sensible pour détecter la pénétration d'iode.

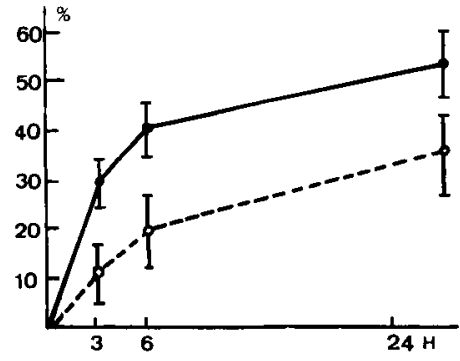

FI G. 2. - Tests de fixation thyroidienne de l'iode 131 obtenus avant application (-) et après application de "Iodex" (- -$)$ pendant 5 jours. Les deux tests pour un même sujet sont effectués à io jours d'intervalle. En ordonnées sont représentés les pourcentages de fixation d'iode I $3 \mathbf{I}$ en fonction du temps suivant l'absorption du traceur (en abscisses); l'expérience a été réalisée sur 9 sujets.

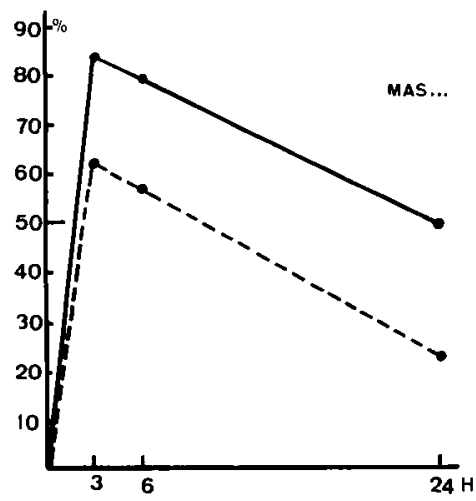

FIG. 3. - Tests de fixation thyoüdienne de l'iode 131 obtenus avant application (-) et apris application de "Iode.x" (- - $)$ pendant 5 jours chez un malade hvperthyroiddien. Les deux tests sont effectués à io jours d'intervalle. On remarquera la franche réduction du taux de fixation et la persistance de l'angle de fuite précoce.

La démonstration d'une baisse de la fixation thyroïdienne est surtout nette dans le cas de l'application d'une pommade à base d'iode (Iodex) ; nous avons noté un abaissement important dans le cas de fixations élevées sans hyperthyroïdie, ce qui confirme bien l'hypothèse d'un accroissement des réserves d'iode secondaires aux applications; enfin, les angles de fuite précoces des hyperthyréoses conservent bien le même aspect mais avec toutefois des valeurs globalement abaissées de la fixation. En ce qui concerne les applications de thyroxine, les diminutions des taux de fixation sont significatives mais moins marquées que précédemment. Il semble néanmoins que la pénétration de l'hormone elle-même s'effectue à un taux relativement élevé compte tenu de la valeur de la dose appliquée $(2 \mathrm{ng})$ puisque l'intensité du freinage est comparable à celui habituellement observé lors de la prise orale, pendant le même temps, de deux comprimés de D-L,-3-5-3' triodothyronine dosés à 25 microgrammes. 


\title{
DISCUSSION
}

Le sodium, tout comme le potassium, pénètre lentement à travers la peau qui lui oppose une sérieuse barrière $\left[\mathrm{I}_{5}\right]$; cette barrière est spécifique du tissu vivant car des fragments de peau isolés et dévitalisés sont beaucoup plus perméables à ces mêmes ions; de même, le passage de l'intérieur vers l'extérieur, testé in vitro, est considérablement plus important que la pénétration classique de sens inverse de ces ions; par ailleurs, on a démontré que le phénomène ne correspondait pas à une simple diffusion mais que la pénétration de chacun de ces ions était compétitive [I3]. L'absorption in vivo de strontium 85 est assez comparable à celle des ions précédents mais nous ne trouvons pas, conformément à WAHLBERG [20], d'absorption initiale rapide du traceur. L'absorption plus élevée de l'iodure I3I est en accord avec les résultats d'autres auteurs $[3,8, \mathbf{1} 4]$; il faut remarquer cependant que le mécanisme de pénétration de l'iodure semble faire intervenir une oxydation intra-épithéliale de l'iodure en iode organique, processus pouvant être responsable de sa meilleure absorption relative et peut-être aussi du caractère continu de celle-ci au cours des bains.

Chez l'Homme, malgré les difficultés d'expérimentation, il nous semble que le pansement occlusif constitue une méthode simple, acceptable et donnant des résultats supérieurs au bain libre d'une portion d'un membre forcément limité dans le temps. Mais l'interprétation de ces tests doit tenir compte du fait que le pansement occlusif modifie les conditions physiques de la pénétration cutanée. L'usage du technétium a permis de mesurer des activités urinaires très appréciables, et ce sans danger pour le sujet.

La démonstration indirecte du passage de substances iodées par freinage de la captation thyroïdienne d'iode I3I atteste de l'intensité du phénomène de pénétration. Certes, il faut faire la part du tôle de l'iode organique contenu dans les préparations et susceptible d'un passage privilégié par rapport à l'iodure, ainsi que de la présence concomitante d'une phase liposoluble (excipient) modifiant les caractères physico-chimiques du passage. Mais nous pensons que cette méthode devrait permettre dans le cas de l'iodure, d'étudier l'influence de nombreux paramètres susceptibles d'agir sur la pénétration telle que la composition du bain, le traitement préalable de la peau, les conditions d'application, etc.

Reçu pour publication en août 1974.

\section{SUMMARY}

\author{
RADIOISOTOPIC METHODS FOR THE STUDY \\ OF PERCUTANEOUS ION ABSORPTION
}

\footnotetext{
Absorption of ions through the skin of a young rat has been studied by measuring the extent of activity in each principal organ in relation to total activity of the radioactive solution bathing the skin. Total activity was also observed in the body stripped of the skin and put into scintilla-
} 
tion crystal. Iodine penetrates more than sodium or potassium, and lasts longer. Absorption of various ions through the human skin has been clearly demonstrated by application of occlusal anti-brachial dressings. Decrease in thyroid uptake rate after application on the skin of a commercial product containing cold iodine or thyroxine is an indirect, but sensitive, method for studying penetration of human skin. Action of different physical parameters which may affect skin absorption of iodine or iodide can also be studied using this method. The major reason for studying ion skin absorption is its therapeutic application and the use of balneotheraps.

\section{RÉFÉRENCES BIBLIOGRAPHIQUES}

[t] Bartek M. J., Labudde J. A., Maibach H. I., I972. Skin permeability in vivo; comparison in rat, rabbit, pig and man. J. Investig. Dermatol., 58, I14-1 23.

[2] Bercovicr J.-P., Mauvais Jarvis P., I97I. Bases physiologiques de l'hormonothérapie stéroïdienne par voie percutanée. Presse Médicale, 38, I675-I678.

[3] Dubarky J.-J., Tamarelle C., Blanguet P., I97I. Mise en évidence de la pénétration percutanée d'électrolytes à l'aide de traceurs radioactifs. Presse Therm. Clin., 108, I-8.

[4] Dubarry J.-J., Blanguet P., Tamarelle C., Dubarry B., i97i. Pénétration percutanée des ions en balnéothérapie thermale. Bord. Méd., 4, 3703-3713.

[5] Malkinson F. D., Rothman S., 1963. Percutaneous absorption, Handbuch der Haut und Geschiechiskrankheiten, Berlin, Springer Verlag, 1 (3), 90-156.

[6] Murray J. L., r969. Thyroïd uptake of iodine I3I from skin exposure. Health Phys., 17, 730-73I.

[7] Ostrenga J., Steinmetz C., Poulsen B., Yette S., i97i. Signifiance of vehicules composition. J. Pharm. Sci., 60, II80-1 I83.

[8] Piatesy P., Vegetti G. P., Rigolisti A., rg66. Transcutaneous absorption of iodine in balneotherapy in relation to recent aquisitions on the mecanism of action of iodine. Ann. Sant. Publica, 27, 1363 - I373.

[9] Pinson E. A., I952. Water exchange and barrier as studied by the use of hydrogen isotopes. Physiol. Rev., 32, 123 .

[io] Rotunno C. A., Villalonga I., Fernandez M., Cereijido M., i97o. The penetration of sodium into the epithelium of the frog skin. J. Gen. Physiol., 55, 7I6-735.

[II] Scheuplein R. J., Blank I. H., I97I. Permeability of the skin. Physiol. Rev., 51, 702-746.

[12] Skog E., WAHLBERG J. E., 1963. A comparative investigation of the percutaneous absorption of metal compounds in the guinea pig by means of the radioactive isotopes. J. Investig. Dermatol., 39, $187-192$.

[i3] Steinbach H. B., I967. Movement of $\mathrm{Na}$ and $\mathrm{K}$ across and within frog skin. Amer. J. Physiol., 212, 37 I-375.

[14] TAS J., FEIGE Y., 1958. Penetration of radioiodode ( $\left.{ }^{131} \mathrm{I}\right)$ through human skin. J. Investig. Dermatol., 30, 193-196.

[15] Tregear R., rg66. The permeability of mammalian skin to ions. J. Investig. Dermatol., 46, I6-27.

[16] Tregear R. T., 1954. Physical functions of skin, London Academic, 1-52, I966.

[I7] Valette G., I954. Les conditions physico-chimiques de l'absorption cutanée. J. Physiol., 46, $52-98$.

[I8] WAHLbERG J. E., I965. "Disappareance measurements "; a method for studying percutaneous absorption of isotope labelled compounds emmetting gamma rays. Acta Derm. (Stockholm), 45, 397-4 4 .

[19] WaflberG J. E., 1965. Some Attempts to influence the percutaneous absorptions rate of sodium $\left({ }^{22} \mathrm{Na}\right)$ and mercuric $\left({ }^{203} \mathrm{Hg}\right)$ chlorides in the guinea pig. Effect of soap, alkyl aryl sulphonate, stripping, and pretreatment with distilled water and nercuric chloride. Acta Derm., 45, 335-343.

[20] Wahlberg J. E., I968. Percutaneous absorption of radioactive strontium chloride Sr $89 . \quad A r c h$. Derm. (Chicago), 97, 336-339. 